EPJ manuscript No.

(will be inserted by the editor)

\title{
Renormalizing Sznajd model on complex networks taking into account the effects of growth mechanisms
}

\author{
M. C. González ${ }^{1}$, A. O. Sousa ${ }^{2}$, and H. J. Herrmann ${ }^{1,3}$ \\ 1 Institute for Computational Physics, Universität Stuttgart, Pfaffenwaldring 27, D-70569 Stuttgart, Germany \\ 2 Systemgestaltung, ETH Zentrum Kreuzplatz 5, F 27.28032 Zurich - Switzerland \\ 3 Departamento de Física, Universidade Federal do Ceará, 60451-970 Fortaleza, Brazil
}

Received: date / Revised version: date

\begin{abstract}
We present a renormalization approach to solve the Sznajd opinion formation model on complex networks. For the case of two opinions, we present an expression of the probability of reaching consensus for a given opinion as a function of the initial fraction of agents with that opinion. The calculations reproduce the sharp transition of the model on a fixed network, as well as the recently observed smooth function for the model when simulated on a growing complex networks.
\end{abstract}

PACS. 89.65.-s Social and economic systems - 89.75.Fb Structures and organization in complex systems - 02.70.Uu Applications of Monte Carlo methods - 07.05.Tp Computer modeling and simulations

\section{Introduction}

Opinions can either be made up by a person or taken over from another person. Sometimes some people try to force their opinions on others. In general, all people are free to form opinions as they see fit. The mechanism of opinion formation is "normative", i.e., normative in the sense of what ought to be, opposed to a "positive" mechanism, which is based on observation what is [1]. Based on this facts, and with the necessary simplifying assumptions, socio-physics gave the opportunity to apply techniques of statistical physics to model opinion formation among people [2 3, 4].

One of the opinion formation models that has generated immediate interest in many authors on the field is the Sznajd model [5], which is based on the slogan "together we stand": Individuals are represented by the lattice nodes (one-dimensiona in its first version), and each randomly selected pair of neighbors convinces all their neighbors of their opinions, if and only if the pair shares the same opinion; otherwise, the neighbors' opinion are not affected. It differs from other consensus models by dealing only with communication between neighbors, and the information flows outward as in rumor spreading: a site does not follow what the neighbours tell the site.

On networks with fixed size, the results of the model do not depend much on the spatial dimensionality and type of neighborhood selected (i.e., two nodes convince the others, three convince the others, etc.) [679]. In the case of $q$ choices of opinion, the model has $q$ homogeneous absorbing states, where all individuals choose the same opinion; in the context of opinion, one says the system reaches consensus. The case of two opinions $(q=2)$ has been the most studied, denoting opinions as Ising variables "up" or +1 , and "down" or -1 . In more than one dimension, the probability $\left(P_{u p}\right)$ of reaching consensus "all up" depends on the initial fraction $p$ of individuals with opinion "up"; for $p>0.5$, the probability of reaching "all up" as stationary state is close to one, while for $p<0.5$ it is negligible, having a sharp transition in $p=0.5$, which can be interpreted as a dynamical phase transition. Computer simulations in [8] indicate that the universality class associated with this dynamical phase transition is different from the universality class of the Ising model. The distribution of time needed to reach the stationary state is a peak followed by a fast decay [10].

Much less is known about the Sznajd model on growing networks. Interactions of groups of people in some circumstances can be thought as a growing system, i.e., in a city with positive rate of immigration. In a first and simple approximation, it can be modeled by a growing scale-free network [11]. Recently, applying a Sznajd model recipe not after the complete network has been constructed, but while the network grows, i.e, while each new node is added to the network, one could observe that the Sznajd model simulated on scale-free networks, Barabási-Albert network and a pseudo-fractal network [12], the system reaches consensus [13 14]. But in contrast to the sharp transition observed for the networks of fixed size, in which the Sznajd recipe is performed only after building up completely the network, the probability that the system reaches "all up" for a growing complex networks is a smooth function of $p$. In addition, this function depends on the type of neighborhood selected.

In this work, we propose a real space renormalization approach [15] to calculate the probability $P_{u p}(p)$ of reaching consensus on opinion "up" as a function of the initial fraction $p$ of opinion "up". Our results are for two common rules of neighborhood, namely " $r$-convince all their neighbors", with 
$r=2$ and $r=3$. We have obtained the two well-known results known for the model: a smooth function of $p$ for the growing case and an expression which approximates the step function for fixed networks.

In the next section, we present the hierarchical network used in our calculations. Then, we present the renormalization approach and the analytical expressions obtained, each case is compared with the results from the numerical simulations, previously reported in [14], as well as for the $B A$ scale-free network.

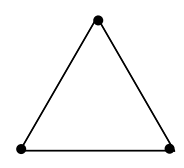

$\mathrm{t}=0$

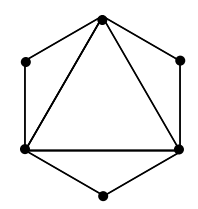

$t=1$

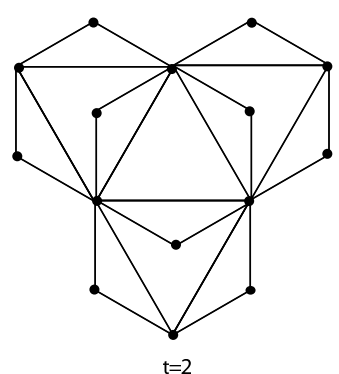

$\mathrm{t}=2$
Fig. 1. The first three generations of the scale-free pseudo-fractal graph. At each iteration step $t$, every edge generates an additional vertex, which is attached to the two vertices of this edge.

\section{Hierarchical Network}

The deterministic scale-free graph used in this work grows as follows: At each time step, every edge generates an additional vertex, which is attached to both end vertices of this edge. Initially, at $t=0$, we have a triangle of edges connecting three vertices, at $t=1$, the graph consists of 6 vertices connected by 9 edges, and so on (see Fig 1). The total number of vertices at iteration $t$ is

$$
N_{t}=\frac{3\left(3^{t}+1\right)}{2}
$$

This simple rule produces a complex growing network. Such a graph is called a pseudo-fractal. In the next section, we present the use of this hierarchical network to find expressions that agree with the simulated results of the Sznajd model on complex networks.

\section{Renormalization Approach}

Our method can be very intuitive and is based on the method proposed by Galam to study bottom-up democratic voting by majority rule in a square lattice [3], where the predictions of the results in all the lattice are based on the applications of the majority rule over a basic cell of neighbors, called renormalization cell.

We find that given a neighborhood rule, it is enough to choose an appropriate generation of a hierarchical network for calculating $\left.P_{u p}(p)\right|_{r, g}$, which agrees with the the numerical results of the model on growing networks The subscript index $r, g$ in $\left.P_{u p}(p)\right|_{r, g}$ is to stress that the resulting function belongs to a chosen Sznajd rule $(r)$ in a growing network $(g)$. Subsequent self-iterations of $\left.P_{u p}(p)\right|_{r, g}$ result in a step function, i.e., $\left.P_{u p}^{n}(p)\right|_{r, g}=P_{u p}(p)_{r, f}$, where the subscript index $f$ correponds to the result obtained for a network of fixed size.

For a population fraction $p$ with opinion "up", the general method is as follows:

- Given a neighborhood rule $r$, the chosen basic cell corresponds to the minimum generation $t$ of the hierarchical network, such that $r>N_{t}$ (the $r$ agents must have at least one agent to convince). We call this resulting number of nodes in the cell $n_{r}$.

- The probability of each possible configuration in a elementary cell is easily calculated, such that

$$
1=\left.P_{\text {all }}(p)\right|_{r}=\sum_{k=2}^{n_{r}} B_{n_{r} k} p^{k}(1-p)^{n_{r}-k} .
$$

with the binomial coefficient $B_{n_{r} k}$ over the appropriate cell for the chosen rule:

$$
B_{n_{r} k}=n_{r} ! /\left[k !\left(n_{r}-k\right) !\right] .
$$

- From all the configurations calculated above, we select the subset that gives "all up" when applying the selected Sznajd rule on the cell, the sum of all of them is $\left.P_{u p}(p)\right|_{r, g}$ :

$$
\left.P_{u p}(p)\right|_{r, g}=\left.P_{\text {all }}(p)\right|_{r, u p}
$$

Next, we illustrate the result of the method with $r=2$ and $r=3$.

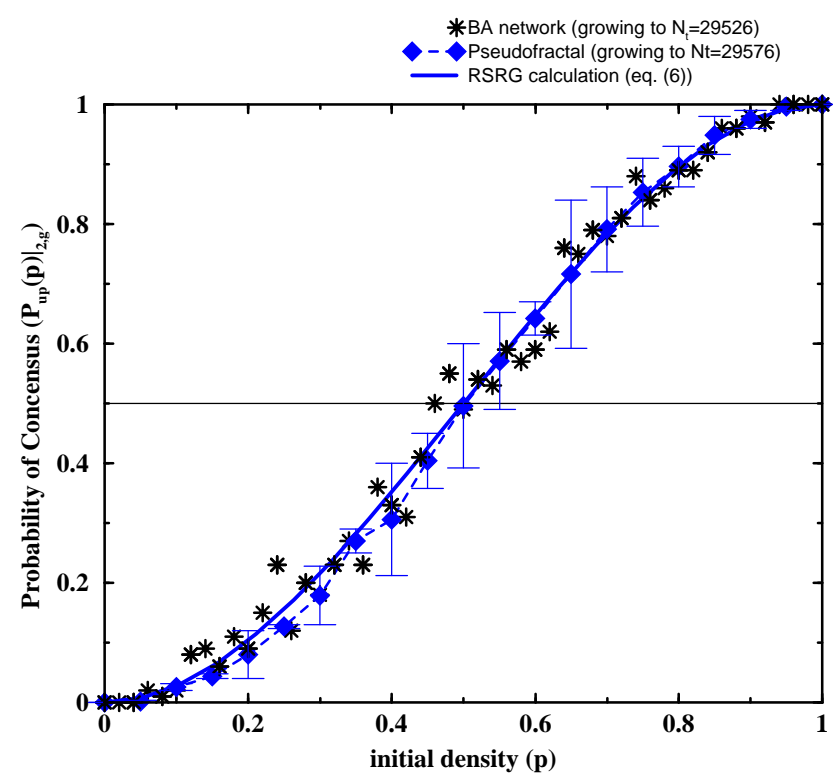

Fig. 2. Comparison between the function presented in Eq. 6 (solid line) with Monte Carlo simulations on a growing pseudo-fractal (triangles with error-bars) and on a growing $B A$ scale-free network (stars). In both networks, 29576 nodes are considered. We count the number of samples, out of 1000 , for which the fixed point all "up" is obtained when different values for the initial concentration $p$ of nodes "up" are simulated for rule $r=2$. 


\subsection{Case $r=2$}

\subsubsection{Growing}

For $r=2$, the triangle of the generation $t=0$ is the basic cell. Thus $n_{r}=3$ and, for a given fraction $p$, all the possible configurations are:

$$
1=\left.P_{\text {all }}(p)\right|_{2}=p^{3}+2 p^{2}(1-p)+2 p(1-p)^{2}+(1-p)^{3}
$$

If we apply the selected Sznajd rule $r=2$ over the triangle, only the configurations expressed in the first two terms of the sum give "all up". Therefore:

$$
\left.P_{u p}(p)\right|_{2, g}=p^{3}+2 p^{2}(1-p)=3 p^{2}-2 p^{3}
$$

In Fig. 22 we can see the good agreement of Eq. 6 with the numerical results [14] for the Sznajd model on a growing pseudofractal, as well as for the Barabási-Albert scale-free network [11].

\subsubsection{Fixed}

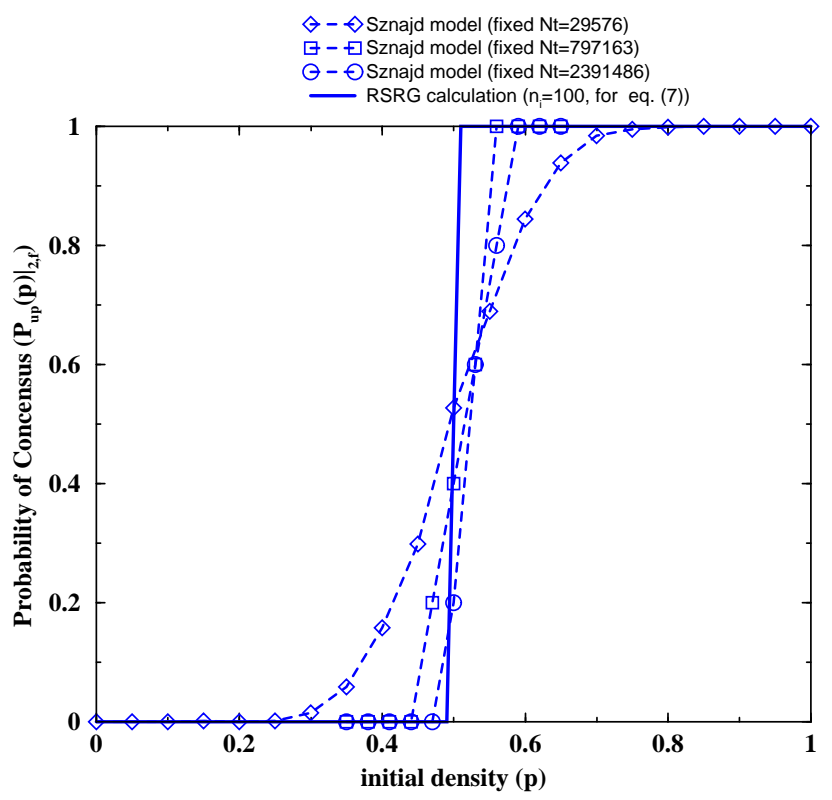

Fig. 3. Eq. 7 with $n_{i}=100$ (solid line) compared with simulations on a fixed pseudo-fractal networks with $N_{t}=29576,797163$ and 2391486 nodes (dashed line with symbols). Other simulation conditions as presented in the caption of Fig. [2]

In order to recover the reported result on a fixed network, one makes renormalization iterations, which means simply selfcomposing the Eq. 6

$$
\left.P_{u p}(p)\right|_{2, f}=\left.P_{u p}^{n_{i}}(p)\right|_{2, g},
$$

and in the limit of large number of iterations $\left(n_{i}-1\right)$, one recovers the step function observed numerically for the model on fixed networks. Note that the number of terms and the coefficients sizes increase very fast, as one can observe in the expression of only one composition:

$\left.P_{u p}^{2}(p)\right|_{2, g}=27 p^{4}-36 p^{5}-42 p^{6}+108 p^{7}-72 p^{8}+16 p^{9}$,

therefore, the multiple compositions presented in Fig. 2 are iterated with a computer. Figure 3 shows that the numerical simulations on large networks tend to the step function calculated from Eq. 8$]$ with $n_{i}=100$.

\subsection{Case $r=3$}

\subsubsection{Growing}

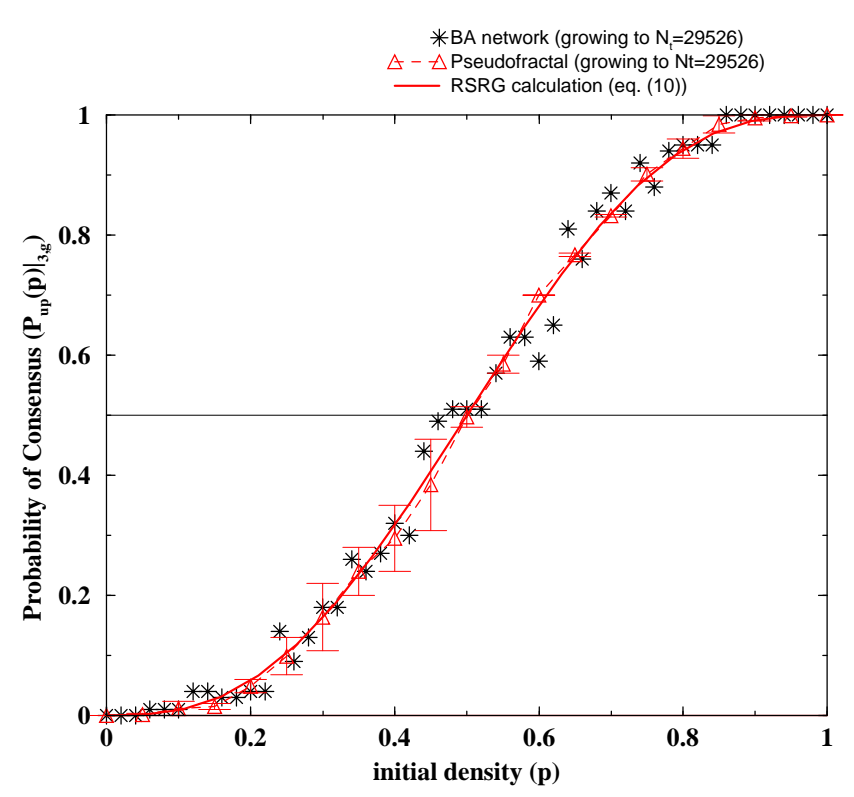

Fig. 4. Eq. 10 (solid line) compared with the results from the simulations on a growing pseudo-fractal (triangles with error-bars) and on a growing $B A$ scale-free network (stars) when $r=3$. The other simulation conditions are the same of Fig. 2

The core of the method is the selection of the correct configurations after applying the Sznajd rule on it. As we will see for this rule, when the number of nodes in the renormalization cell is even, there are some symmetrical configurations which can have either "all up" or "all down" with the same probability. In this case only half of them are summed to $P_{u p}$. For $r=3$, the generation $t=1$ is the basic cell. Thus $n_{r}=6$ and, for a given fraction $p$, all the possible configurations are:

$$
1=\left.P_{\text {all }}(p)\right|_{3}=(1+(1-p))^{6} .
$$

Note that the values of the binomial coefficient in the consecutive terms are: $1,6,15,20,15,6,1$. From the 20 configurations of the $4 t h$ term, there are 7 that give "all up"(shown in Fig. 6 at Appendix 44, the corresponding 7 opposed cases which give "all down", and 6 symmetrical configurations shown in Fig. 7 
(Appendix 44 that can give either "all up" or "all down". Therefore, these group of configurations contribute with $7+0.5 \times 6$, and we have:

$\left.P_{u p}(p)\right|_{3, g}=p^{6}+6 p^{5}(1-p)+15 p^{4}(1-p)^{2}+10 p^{3}(1-p)^{3}$

In Fig. 4 we see that Eq.10 agrees very well with the numerical results [14] for the Sznajd model on a growing network when the rule $r=3$ is considered.

\subsubsection{Fixed}

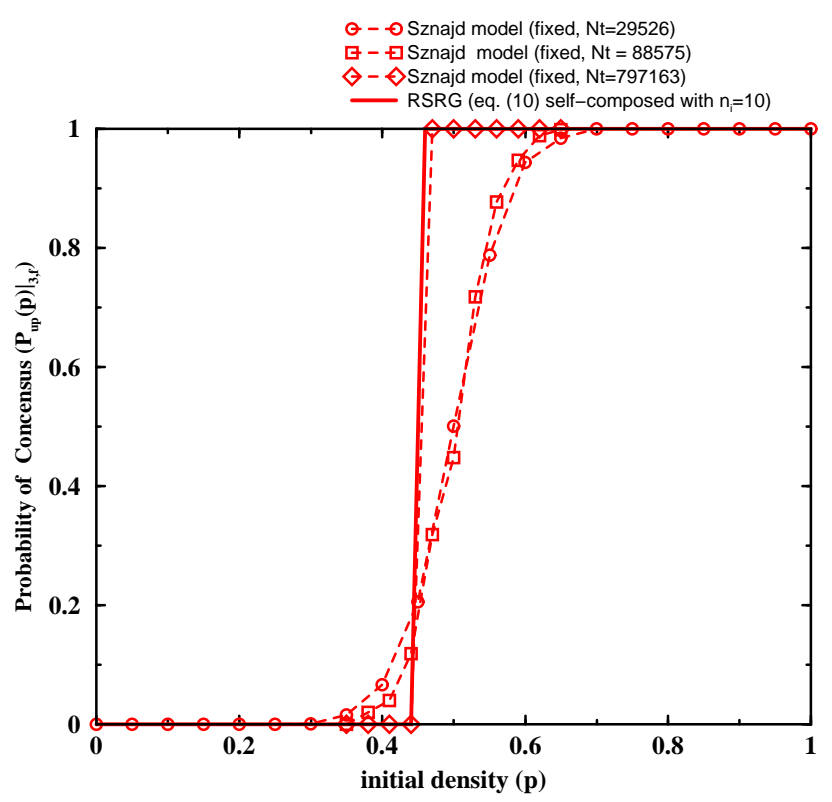

Fig. 5. Eq.10 self-composed in 9 steps (solid line) compared with the result from the simulations on a fixed pseudo-fractal networks with $r=3$ (dashed lines with symbols). The other simulation conditions are the same as presented in the caption of Fig. 3

The result of the composition for this case is far more complicated and only 1 self-composition of eq.10 $\left(n_{i}=2\right)$ already needs a computer, as shows the following expression:

$$
\begin{gathered}
\left.P_{u p}(p)\right|_{3, g} ^{2}=-1249989 p^{12}+390897 p^{11}-158184 p^{10}+28561 p \\
643783179 p^{18}+270741222 p^{17}-100735317 p^{16}+41109081 \\
p^{15}-17504838 p^{14}+5585931 p^{13}-15244686567 p^{24}+ \\
11863411551 p^{23}-7642674243 p^{22}+4315583718 p^{21}- \\
2347570026 p^{20}+1281132990 p^{19}-816731505 p^{30}+ \\
2281401855 p^{29}-5100164190 p^{28}+9199907505 p^{27}- \\
13440029166 p^{26}+15908268375 p^{25}-2187 p^{36}+65610 p^{35} \\
-925101 p^{34}+8148762 p^{33}-50268195 p^{32}+230706630 p^{31}
\end{gathered}
$$

In Fig. 5 we see the step function obtained with only 9 steps of composition compared with the numerical results on a fixed network of different sizes; as we see the results agree very well with the simulations of the model on large networks.

\section{Conclusions}

Based on opinion formation rules of the usual Sznajd model, we use a renormalization approach to give an expression for the probability of consensus into one opinion as a function of the initial fraction of this opinion.

We show that for a given Sznajd rule it is enough to solve exactly the model on an appropriate basic cell in order to find an expression for the smooth function, found numerically for the model on a growing network. Several self-compositions of the obtained function give the step function observed for the model on a network of fixed size. Further renormalization patterns has to be tested, but in order to reproduce the results of the Sznajd model on growing $S F$ networks, a $S F$ hierarchical network must be chosen.

The proposed method could be, in principle, extended to other types of neighborhood and more interestingly to many choices of opinion $(q>2)$, which is an feature of the model used to simulate elections processes [16 14 10], obtaining results consistent with some empirical observations [17].

\section{Acknowledgments}

The authors would like to thank Dietrich Stauffer and Katarzyna Sznajd-Weron for useful discussions. MCG thanks Deutscher Akademischer Austausch Dienst (DAAD), Germany, for financial support.

\section{Appendix A: Configurations with the same fraction of nodes "up" and "down"}

Here we present some of the possible configurations applying the Sznajd rule corresponding to the $r=3$ on its appropriate renormalization pattern $\left(n_{3}=6\right)$. In particular, we show the case of half of the nodes having opinion up, mentioned in Section 3.2.1 and represented by the fourth term in Eq.10

Figure 6 shows the 7 configurations that give as a result "all up", when applying the Sznajd rule, i.e., three consecutive nodes with opinion +1 convince all their neighbors. Note that interchanging + and - , we have the 7 configurations for the opposed case of consensus "all down".

Figure7presents the 6 symmetrical configurations that have 93 consecutive nodes with +1 , as well as 3 nodes with -1 giving consensus "all up" or "all down", respectively. Thus, these configurations contribute wit $0.5 \times 6$ to the probability of consensus "all up" $\left(P_{p}\right)$, as showed in Section 3.2.1

\section{References}

1. http://en.wikipedia.org/wiki/Opinion

2. D. Stauffer, Journal of Artificial Societies and Social Simulations 5, issue 1, (jass.soc.surrey.ac.uk) (2002); D. Stauffer, S. Moss de Oliveira, P.M.C. de Oliveira, J.S. Sa Martins, Biology, Sociology, Geology by Computational Physicists, Elsevier, Amsterdam (2006) in press.

3. S. Galam, Physica A, 28566 (2000).

4. S. Galam, Eurphys. Lett., 70705 (2005). 


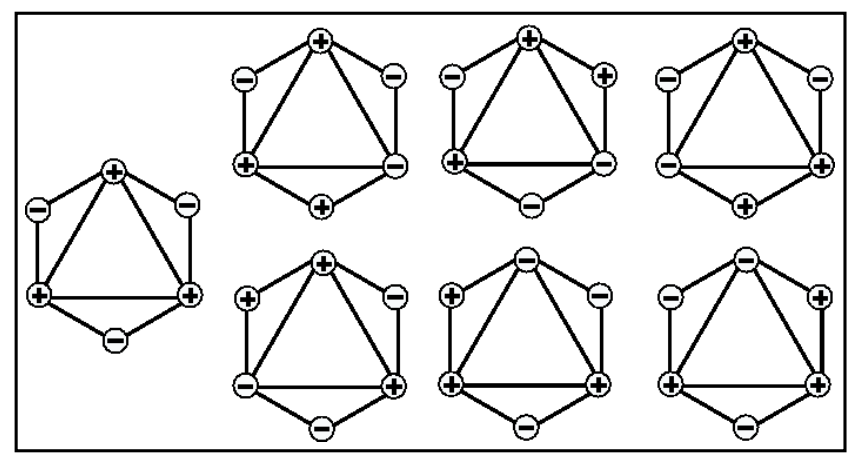

Fig. 6. Configurations that generate consensus "all up" with $r=3$ and the same fraction of opinions "up" and "down".

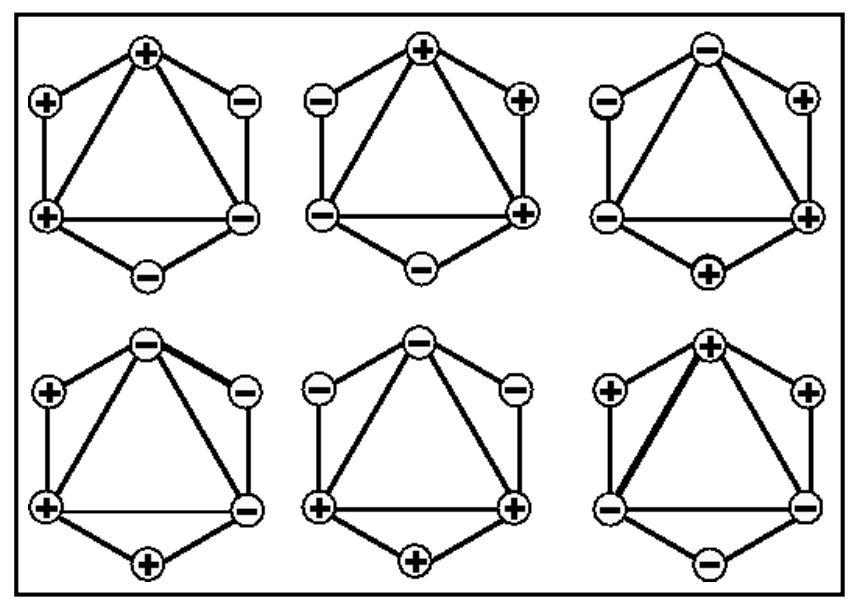

Fig. 7. Configurations that generate either consensus "all up" or "all down" with $r=3$.

5. K. Sznajd-Weron and J. Sznajd, Int. J. Mod. Phys. C 11, 1157 (2000).

6. D. Stauffer, A.O. Sousa, and S. Moss de Oliveira, Int. J. Mod. Phys. C 11, 1239 (2000).

7. A.O. Sousa, Physica A 348, 701, (2005).

8. D. Stauffer and P.M.C. de Oliveira, Eur. Phys. J. B 30, 587 (2002).

9. C. Schulze, Int. J. Mod. Phys. C 15, 867 (2004)

10. F. Slanina and H. Lavička, Eur. Phys. J. B 30, 587 (2002).

11. R. Albert and A.-L. Barabási Rev. Mod. Phys. 74, 47 (2002).

12. S.N. Dorogovtsev, A.V. Goltsev and J.F.F. Mendes, Phys.Rev. E 65, 066122 (2002).

13. J. Bonnekoh, Int. J. Mod. Phys. C 14, 1231 (2003).

14. M.C. González, A.O. Sousa, and H. J. Herrmann, Int. J. Mod. Phys. C 15, 45 (2004).

15. P.J. Reynolds W. Klein and H. E. Stanley, J. Phys C 10 L167 (1977).

16. A.T. Bernardes, D. Stauffer, and J. Kertész, Eur. Phys. J. B 25123 (2002).

17. R.N. Costa Filho, M.P. Almeida, J.S. Andrade, Jr., and J.E. Moreira, Phys. Rev. E., 60, 1067, (1999). 